\title{
A Smart Chaos Managing Cum Energy Saving System for Hospital
}

\author{
Divya B ${ }^{1}$, Remya George ${ }^{2}$, Mr. Jis Paul ${ }^{3}$, Binoy John ${ }^{4}$, Kochuthresia Jose ${ }^{4}$, \\ Muhammed Basheer ${ }^{4}$, Nisanth T M ${ }^{4}$, Sherin Mathew ${ }^{4}$
}

\begin{abstract}
This paper presents a surveillance and energy saving system for a Hospital. The major wastage occurring in the hospitals are human energy (chaos \& efforts from staff to manage crowd) and electrical energy. So the idea is to develop a smart chaos managing system integrated on the present surveillance system that automatically detects and alerts the security personals and thus will reduce the extra efforts of medical staff. Along with this a virtual electrical monitoring is present which can detect and identify short circuits and other problems and also turn off lightings and fans in places such as offices. This in total will be monitoring and automatically detecting chaos and directing securities and control electrical energy wastage in hospitals.
\end{abstract}

Keywords: Image Processing, PIR sensor, Signal processing, Zigbee.

\section{Introduction}

Many hospitals and health clinics use surveillance system to take care of the people security and ensure that the patients will receive proper care. Electronic medical devices provide a vast array of medical information while allowing the patient to have a user-friendly interface. This trend in medical technology towards easier and more effective tools for health care ultimately leads to a huge amount of energy loss and thus will affect the productivity of a hospital. Nurses and security guards represent roughly one-third of workers in hospitals. Approximately 60 percent of nurses and security guard in the hospitals are estimated working up to 10 hours of overtime each week due to time wasted trying to manage and control people and to locate patients and communicate with staff. Our project focuses on the detection and controlling chaos occurring in the hospital premises and managing the energy wastage in hospital environment. This work aims to develop a monitoring system for hospitals, to determine chaos in front of Operation Theater or similar places by examining the sound level and observing through a surveillance camera.

\section{Back Ground}

People are of different kind in our society, so controlling them is a very tedious task and requires extra efforts of time and energy. But in places like hospitals it is highly recommended for the ambient condition to maintain. Nurses and security guards represent one-third of workers in hospitals. Nurses face a variety of problems caused by communication challenges. A major problem is wasted time in controlling people in front of areas like ICUs and critical care units. The OP and pharmacy section is more prone to chaos (crowding, shouting and fights). So the solution should be in such a way to detect and direct the security personals to problem site anywhere within the hospital premise.

One other problem faced by the hospital is the electrical energy wastages. Issues like short circuits leads to power failures will highly affect people subjected in the intensive care units and usually it goes to large delay in identifying and rectifying problem. Also in official rooms such as the financial sections face a problem of lights and fans switching mostly people forget and a huge amount of energy gets wasted. So the solution should to be to map the electrical wiring of the hospital and to virtually monitor entire hospital wiring through a system console.

\section{Project Idea}

This project aims to develop a monitoring system for hospitals, to determine chaos in front of Operation Theater or similar places by examining the sound level and observing through a surveillance camera. The project will be having two sections, an embedded system with wireless camera, servo motor, relay, current sensor, human detection sensor(PIR) etc interfaced to a microcontroller and a user interface application (Matlab) system running on a system/laptop .

A wireless camera is used to transmit the images and sound to a computer system where the data received is processed using Matlab. When the sound level increased beyond a particular value and the image will be processed and made to detect any change in the normal pattern and found that the number of persons is greater than a particular value, alarm is triggered. 
1. Mrs.Divya B, Asst Professor, Biomedical, Calicut University, SCET, Thrissur, India, Email:divyadas866@gmail.com

2. Mrs.Remya George Biomedical, Calicut University, SCET, Thrissur, India , E-mail :remyageorge83@gmail.com

3. Mr. Jis Paul, Asst Professor, Biomedical, Calicut University, SCET, Thrissur, India, Email:jispaul79@gmail.com

4. Binoy John, Kochuthresia Jose, Muhammed Basheer, Nisanth T M, Sherin Mathew, Students, Sahrdaya College of Engineering and Technology, Thrissur, India

The system incorporates a human detection sensor (PIR) to determine the presence or absence of humans, a relay system to shut down the power in the absence of humans, a servo motor to rotate the camera and a current measurement circuit to detect short circuit.

In a Hospital the user can control the power and camera angle using a user interface in the system/laptop. Zigbee module will be used for the communication between the system and the microcontroller. Finally it will facilitate viewing of live and recording images and does the real time processing and hence will help in managing the chaos and save energy. The output section contains a (GUI) graphical user interface for the extra function and features available on the monitoring screen. There will be a storage device for recording and storing the videos captured. The system will also be ensuring that recorded videos cannot be altered and stored properly for evidential purpose. The datas acquired will be stored for later documentation in the system which can be accessed easily anywhere in the area.

\section{I Technical Knowledge}

- $\quad$ Embedded C programming

- Matlab programming

- Zigbee Communication

- RF camera interfacing

- Relay interfacing

- PIR sensor interfacing

\section{System Design}

The conceptual block diagram of the system was developed which consist of a master console and a slave console. The master console includes a graphical user interface through which the security personal can interact with the system. A servo motor affixed on the camera enables the movement of camera for proper surveillance. Slave console integrate the audio video as well as the PIR. The current voltage as well as the relay switching is controlled by the processor.

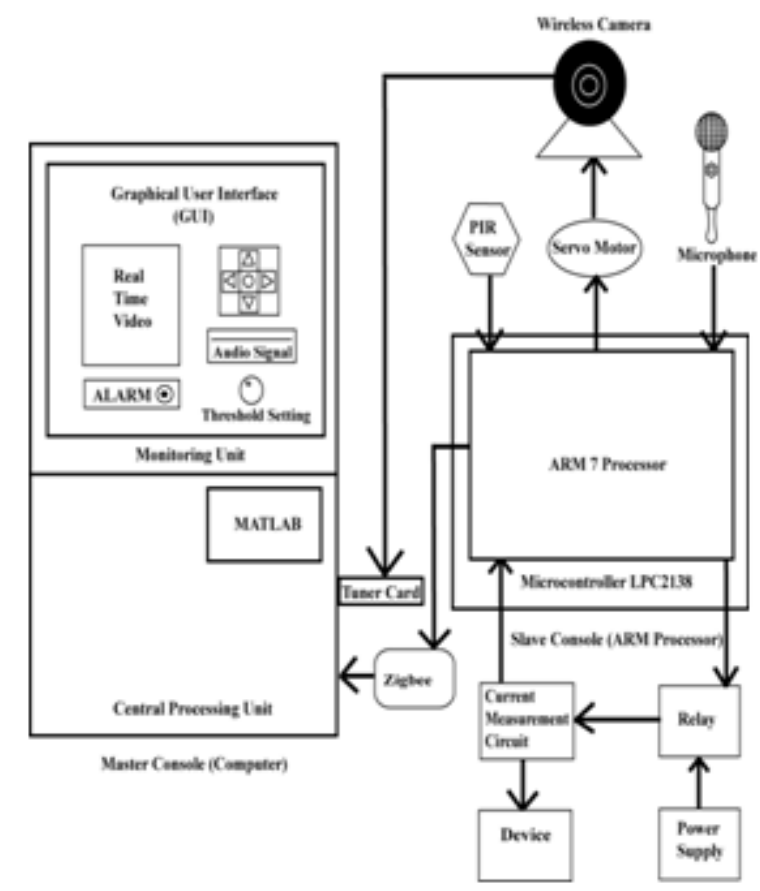

Fig 2: Block Diagram 


\subsection{Embedded System}

The brain of the embedded system part can be developed on a 32bit microcontroller with ARM7 processor (LPC2138). The programming of this controller is done using C language. Servo motor, PIR sensor, current measurement circuit and Zigbee module are attached to the peripherals of the micro controller.

The LPC2138 micro controllers are based on a 32 bit ARM7TDMI-STM CPU with real-time emulation and embedded trace support, that combines the $64 \mathrm{kB}$, of embedded high speed Flash memory. Due to their tiny size and low power consumption, these micro controllers are ideal for applications where miniaturization is a key requirement. With a wide range of serial communications interfaces and on-chip SRAM options of $32 \mathrm{kB}$, they are very well suited for this work.

\subsection{System Software}

System software for sound and picture can be developed using Matlab. The system software will be accessing video and audio port of the system. This software displays the image send by the wireless camera and transmits user controls to the embedded system.

\subsection{Zigbee Communication}

Zigbee is a low-cost, low-power, wireless mesh networking standard. First, the low cost allows the technology to be widely deployed in wireless control and monitoring applications. Second, the low power-usage allows longer life with smaller batteries. Third, the mesh networking provides high reliability and more extensive range. The standard takes full advantage of the IEEE 802.15.4 physical radio specification and operates in unlicensed bands worldwide at the following frequencies: $2.400-2.484 \mathrm{GHz}, 902-928 \mathrm{MHz}$ and $868.0-$ $868.6 \mathrm{MHz}$.

\subsection{Human Detection (PIR)}

One of the aims of the project is to save the energy .Intelligent Energy Saving System can be used in places like where lighting is very important. The hospital rooms will be well illuminated with many lamps and fans or AC. When people are not present at a room the lighting and other appliances can be made OFF and when they are present, the lighting made ON. All these can be done through by a PIR sensor

\subsection{Current And Voltage Detection}

The current flowing through the wire is detected and monitored by keeping a CT-coil surrounding the wire. The CT-coil works by Faraday's law of electromagnetic induction. Thus, any change in the intensity of current flowing across the wire induces an emf in the coil proportional to the change in current intensity. Thus from the emf output of CT-coil any short circuits, line breakages etc., can be detected without the need of human intervention.

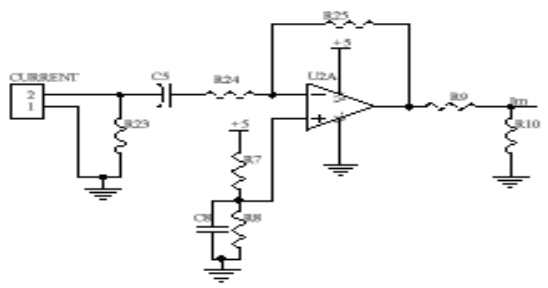

Figure 4: Circuit Diagram for Current Detection

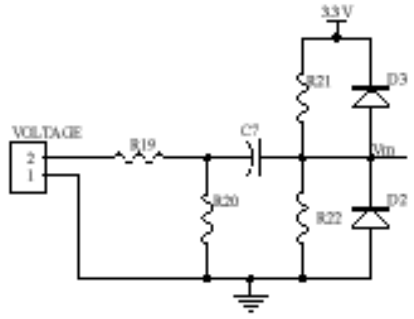

Figure 5: Circuit Diagram for Sound Detection

\subsection{Mean Shift Algorithm}

The mean shift algorithm is used for visual tracking. The simplest such algorithm would create a confidence map in the new image based on the color histogram of the object in the previous image, and use mean shift to find the peak of a confidence map near the object's old position. A few algorithms, such as 
ensemble tracking expand on this idea. It is based on probability density functions, ensembles and iterations. In our program we are using these steps given below for the tracking of chaos.

1. Compute mean shift vector

2. Move the density estimation window

3. Repeat till convergence

\subsection{Audio System}

The use of a sound detection and recognition system can offer concrete potentialities for surveillance and security applications, by contributing to alarm triggering or validation. Furthermore, these functionalities can also be used in portable tele-assistive devices, to inform disabled and elderly persons affected in their hearing capabilities about relevant environmental sounds.

The on-line surveillance system, is made up of a microphone recording the sound activity. Whenever the detection module is finding discontinuities or anomalies in the input signal, the recognition process is activated. A time-frequency analysis of the signal is then performed, and the class of the detected sound is determined after comparison with different sound models, trained from a database. Adequate human intervention (eg. intervention patrols, fire brigade, etc.) can then be undertaken according to the automatic system verdict.

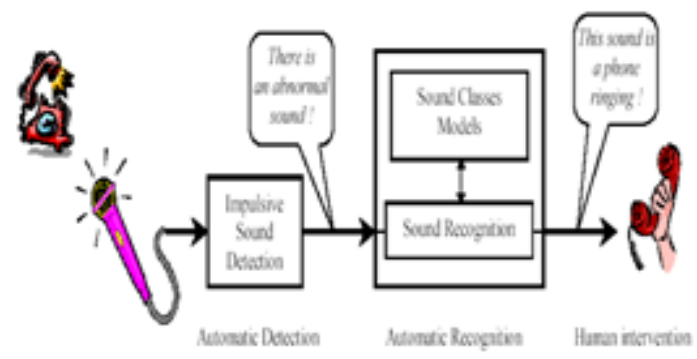

Fig5: Block Diagram of Audio System

\section{Results:}

After developing and integrating the system we could obtain the real-time video of the area where the camera is installed. Then we take the current, voltage and human presence information in the area and transmitted the information efficiently using the zigbee to the master console where the security personal can monitor the scene. All the transmissions and receptions of signals were successfully achieved through zigbee interfacing. In addition the slave console could also effectively turn on/off the electrical appliances in the area based on the human presence as detected by the PIR module which in turn controlling the relays. The snap shot of the entire hardware which we developed is shown below.

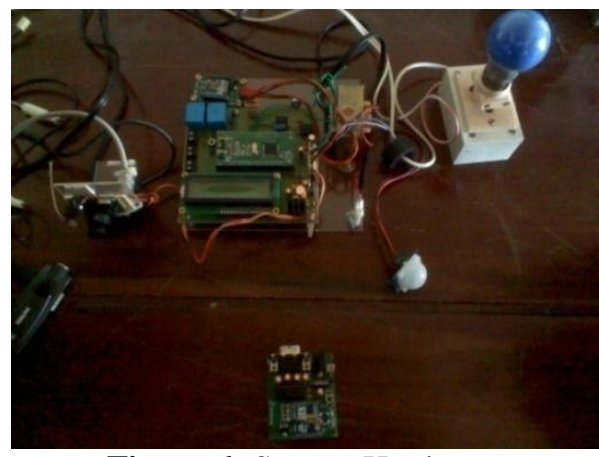

Figure 6: System Hardware

The GUI which we developed consisted of two display panels, one for audio and one for video monitoring. There were two push buttons in the GUI. After pressing the 'Start' buttons the audio video capturing starts in our system. The system will start acquiring snap shots as well as audio sample which will be displayed on the GUI. In addition to this there is a servo control section for camera movement. Based on the requirement of the person it can be shifted to right or left. There are different message displays box for current voltage and other messages in the GUI. The snap shot of the entire GUI which we developed is shown below. The system could accurately transfer any control signals send from the master console to the slave console kept 
in the preferred area (in the hospital) for instance any camera shift given through the master console was efficiently received at the slave console and the camera moved accordingly

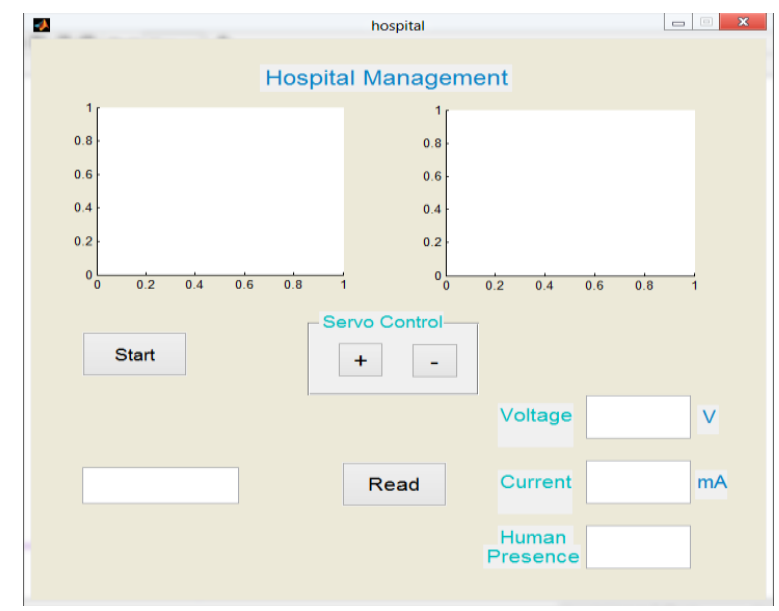

Figure 7: Master Console

After developing the entire system and integrating the testing of the system was done. We took the class room as the area to be tested. The camera was affixed on platform from which a large field of view is obtained for the room. Initially a sample with ambient sound condition and snap shot without any crowd is taken and stored as reference. Then the system is started by pressing the 'Start' button the image capturing takes place immediately. The audio waveform of the room can be seen on the right display.

If audio has crossed the threshold and the video is having a high density of people the condition for chaos has been satisfied and hence the message box will display 'CHAOS' and give alert signals through zigbee communication. Meanwhile the present reading of the appliance connected on to the system is displayed on the message boxes. Finally the presence of human is displayed after checking it with the PIR sensor

\section{Conclusions}

Finally with the successful completion of the project, it will be an efficient system that reduces the extra efforts of the security personals and supporting staffs in managing different resources in a hospital and thus saves time and energy. Thus, it not only reduces the work of the nurses and supporting staff, it also reduces the expenditure of the hospital in terms of over-time expenditure, repair of power-lines and equipments that could have been salvaged from short circuit and also clear the disorder and general chaos caused in hospitals due to the large influx of visitors, indirectly providing peace and comfort to the patients. With further research and innovation, this project can be implemented in different areas other than hospital management; like security surveillance, malls, textiles and nuclear power plant safety. We also plan to integrate software in the GUI which outlines the electrical circuit of different rooms in a hospital, so that we are able to directly test the different lines of the circuit in the GUI itself instead of going to the room and checking it manually

\section{Acknowledgment}

Almighty God was always with us, giving the hope and courage to accomplish the task in time. We express our sincere thanks to Almighty from the depth of the heart. We are deeply indebted to our Head of the Department Mr. Jis Paul for filling us with a lot of ideas and extending all helping hand during this course of time. We extend our deep sense of gratitude to our Project Guide Mrs. Divya B for her whole hearted support and guidance and also to our project coordinator Mrs Remya George for providing her enlightening guidance throughout the project with encouragement and support.

\section{References}

[1] S. M. Metev and V. P. Veiko, Laser Assisted Microtechnology, 2nd ed., R. M. Osgood, Jr., Ed. Berlin, Germany: Springer-Verlag, 1998.

[2] J. Breckling, Ed., The Analysis of Directional Time Series: Applications to Wind Speed and Direction, ser. Lecture Notes in Statistics. Berlin, Germany: Springer, 1989, vol. 61.

[3] S. Zhang, C. Zhu, J. K. O. Sin, and P. K. T. Mok, "A novel ultrathin elevated channel low-temperature poly-Si TFT," IEEE Electron Device Lett., vol. 20, pp. 569-571, Nov. 1999.

[4] Jian-Shuen Fang et al, "Real-time human identification using a pyroelectric infrared detector array and hidden Markov models", optics express 6643, vol.14, pp.15, July 2006.

[5] Mr. S.P Vijayaragavan et al, "Live Human Detecting Robot for Earthquake Rescue Operation", International Journal of Business Intelligents, Vol 02, Issue 01, June2013 